\title{
Determinación de riesgo cardiovascular y edad vascular según el score de Framingham en pacientes del Hospital Nacional Arzobispo Loayza
}

Jean Carlo Mayta Calderón ${ }^{1}$, Adriana Mercedes Morales Moreno ${ }^{1}$, Alejandra Denisse Cárdenas Rojas ${ }^{1}$, J ulissa Ángella Mogollón Lavi ${ }^{1}$, Victoria Armas Rodríguez², Luis Neyra Arismendiz ${ }^{3}$, Carlos Enrique Ruíz Mori ${ }^{4}$

\section{RESUMEN}

Las enfermedades cardiovasculares ocupan el cuarto lugar de carga de enfermedad en Perú, y en los últimos 7 años, la población de alto riesgo cardiovascular se ha incrementado.

Objetivo: Determinar el riesgo cardiovascular y edad vascular según el score de Framingham de los pacientes del Hospital Nacional Arzobispo Loayza así como determinar su factor de riesgo más prevalente. Y las características clínicas de los pacientes con mediano y alto riesgo.

Material y Métodos: Estudio descriptivo, observacional, transversal. Se encuestaron a 238 pacientes hospitalizados en el Servicio de Medicina Interna del hospital. Se consideró: edad, género, diabetes, tabaquismo, IMC y presión arterial. Los datos fueron analizados con el programa SPSS v. 21.

Resultados: Se encontró que el mayor porcentaje de la población de estudio presentó mediano y alto riesgo, siendo el factor más prevalente la diabetes y la mayoría hombres. La diferencia entre la edad cronológica y la edad vascular fue en promedio 6,9 años.

Conclusión: La población estudiada tuvo mediano y alto riesgo por lo que se deberían tomar medidas de prevención primaria y secundaria. (Horiz Med 2015; 15(2): 27-34)

Palabras clave: riesgo cardiovascular, edad vascular, enfermedad cardiovascular, Score de Framingham. (Fuente: DeCS BIREME).

Determination of cardiovascular risk and vascular age by the Framingham score in patients at the Arzobispo Loayza Hospital

\section{ABSTRACT}

Cardiovascular diseases rank fourth place of disease burden in Peru, and in the last 7 years, the population of high cardiovascular risk has increased.

Objective: Determine the cardiovascular risk and vascular age according to the Framingham score in patients attending the "Hospital Nacional Arzobispo Loayza", as well as to determine the most prevalent factor. Furthermore, to determine the clinical characteristics of patients with mild and high risk.

Material and Methods: This is a descriptive, observational, cross-sectional study. 238 hospitalized patients in internal medicine wards were surveyed. Variables such as age, gender, diabetes, smoking, BMI and blood pressure were considered. The data were analyzed using SPSS v. 21.

Results: The highest percentage of the population was found within the mild and high risk categories, with diabetes being the most prevalent factor. The average difference between chronological age and vascular age was 6.9 years.

Conclusion: The majority of the population has mild and high risk, thus primary and secondary prevention measures should be taken. (Horiz Med 2015; 15(2): 27-34)

Key words: cardiovascular risk, vascular age, cardiovascular disease, Framingham Score. (Source: MeSH NLM).

\footnotetext{
Alumnos de Medicina Humana de la Universidad de San Martín de Porres. Lima, Perú

Jefe del Servicio de Cardiología Hospital Nacional Arzobispo Loayza (HNAL). Lima, Perú

Servicio de Endocrinología Hospital Nacional Arzobispo Loayza (HNAL). Lima, Perú

Jefe del Servicio de Cardiología Instituto Nacional de Enfermedades Neoplásicas. Lima, Perú
} 


\section{INTRODUCCIÓN}

Desde comienzos del siglo $\mathrm{XX}$, las enfermedades no transmisibles (ENT) han sido vistas como una epidemia debido a que la morbilidad y la mortalidad han ido en aumento y cada vez a edades más tempranas. En el 2008, las ENT causaron más del $60 \%$ de muertes a nivel mundial (1).

Dentro de ellas se encuentran las enfermedades cardiovasculares (ECV); grupo de patologías que incluyen enfermedades:isquémicas del corazón, cerebrovasculares y patología de los vasos sanguíneos como: hipertensión y enfermedad vascular periférica. Principal causa de muerte prematura en el mundo ocupando la cardiopatía isquémica el primer lugar y la enfermedad cerebrovascular, el tercero (2).

Estas enfermedades son fundamentalmente producto de la ateroesclerosis, de origen multifactorial. Se han descrito varios factores de riesgo asociados, algunos de ellos, no modificables como la edad o el sexo y otros modificables como tabaquismo, obesidad, sedentarismo, dieta rica en grasas, diabetes, hipertensión arterial entre otros. La mayoría con más de un factor de riesgo siendo necesario intervenir sobre estos factores para prevenir los eventos cardiovasculares anteriormente descritos (3).

En este contexto, y se han establecido escalas para determinar el riesgo cardiovascular en relación a la combinación de los factores de riesgo como: PROCAM, SCORE, REGICOR o la Escala de Framingham la cual ha sido la más difundida y utilizada en Latinoamérica. Permite determinar el riesgo de padecer cualquier evento cardiovascular en 10 años, valorando factores como: edad, sexo, índice de masa corporal, presión arterial, diabetes y tabaquismo, asignando un puntaje a cada uno de ellos y estratificando al paciente en bajo, mediano $\mathrm{y}$ alto riesgo cardiovascular.

Permite además, calcular la edad vascular, concepto relativamente nuevo pero que nos da una estimación del daño vascular del paciente, mediante la variación de años entre esta y su edad cronológica (4).
Ruiz Morí y colaboradores, encontraron en los últimos 7 años que la población de alto riesgo se ha incrementado, afectando más a los hombres que a las mujeres, debido a un estilo de vida desfavorable para la salud vascular.

El alto riesgo cardiovascular es mayor en la costa, que en sierra o selva; y son las ciudades con mayor desarrollo económico y urbanístico donde el riesgo cardiovascular se incrementa marcadamente (5).

El Hospital Nacional Arzobispo Loayza (HNAL) es del Ministerio de Salud y la mayoría de sus pacientes pertenecen a un estrato socioeconómico bajo.

El presente estudio, pretende determinar el riesgo cardiovascular de los pacientes hospitalizados mediante el Score de Framingham y a su vez, determinar las características clínicas que nos permitan generar un perfil del paciente por categoría de riesgo para posteriormente tomar medidas preventivas. Asimismo, se buscó determinar la edad vascular y la diferencia entre la edad cronológica y la edad vascular (delta) de tal manera que estimemos el daño vascular de la población en estudio.

\section{MATERIAL Y MÉTODOS}

Estudio descriptivo, observacional, transversal. La población estuvo constituida por los pacientes hospitalizados en el servicio Medicina Interna del HNAL en el mes de Febrero 2014 que fueron seleccionados y cumplían con los criterios de inclusión. Criterios de inclusión: pacientes entre 30-74 años. Se excluyó a todo el que haya tenido algún evento cardiovascular previo como: infarto al miocardio, insuficiencia coronaria, angina, accidente cerebrovascular isquémico, accidente cerebrovascular hemorrágico, ataque isquémico transitorio, claudicación intermitente o insuficiencia cardiaca.

Para obtener el tamaño de la población hospitalizada durante un mes en el HNAL se recurrió a información brindada por el área de estadística del HNAL. Se calculó el promedio de pacientes que se hospitalizaron por mes en el Servicio de Medicina 
Interna durante el periodo enero- diciembre 2013, encontrándose que durante un mes se hospitalizaron un promedio de 428 pacientes tomándose este número como la población total.

El tamaño de la muestra fue obtenida mediante la fórmula para calcular parámetros, obteniendo una muestra de 196 pacientes.

Se realizó el tipo de muestreo aleatorio sistemático, obteniéndose un total de 273 encuestas, eliminándose 35 encuestas por no cumplir con los criterios de inclusión y exclusión, quedando un total de 238 encuestas siendo resultados extrapolables a la población. (Figura1)

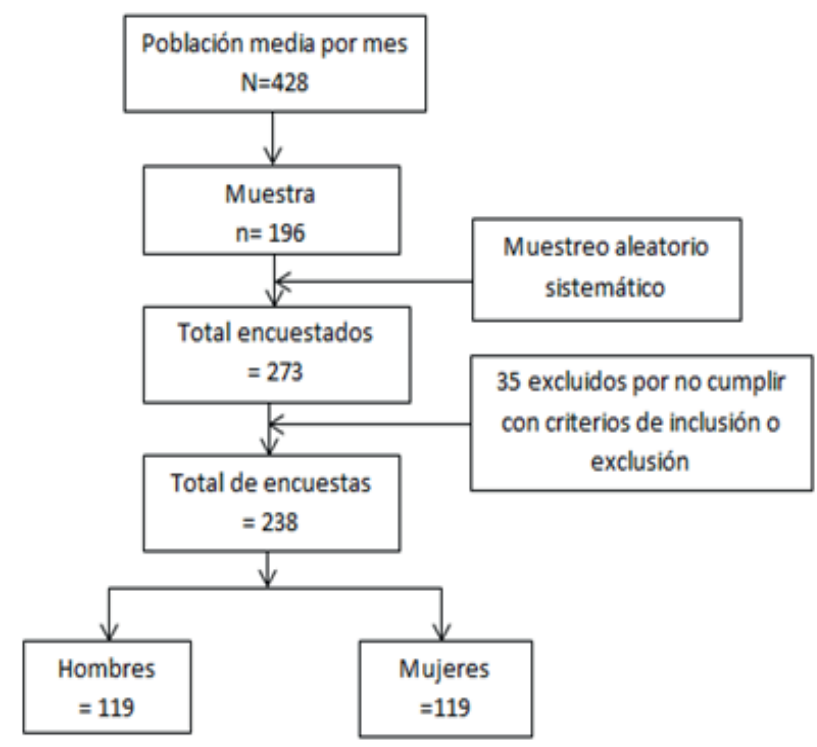

Se consignaron las variables: edad, sexo, tabaco, diabetes, IMC y presión arterial sistólica, riesgo cardiovascular, edad vascular y delta (edad vascular-edad cronológica).

En relación al tabaco, se utilizó la Guía Nacional de abordaje Técnico al Tabaquismo 2010 Perú (6), en la cual se considera fumador a aquella persona que ha fumado al menos un cigarrillo en los últimos seis meses y no fumadora a aquella que nunca ha fumado o ha fumado menos de 100 cigarrillos en toda su vida.

La edad vascular, se definió como la edad de otra persona con el mismo riesgo CV pero con los factores de riesgo en niveles normales (PAS no tratada de $125 \mathrm{mmHg}$, colesterol total $180 \mathrm{mg} / \mathrm{l}$, HDL de $45 \mathrm{mg}$ $\mathrm{dl}$, no fumador, no diabético (7), que se obtuvo por la calculadora del Framingham Heart Study (12).

Presión arterial sistólica; definida como la presión máxima que se alcanza en la sístole. Esta depende fundamentalmente del débito sistólico, la volemia y la distensibilidad de la aorta y las grandes arterias. Se utilizó la clasificación de presión arterial de la JNC VII la cual considera como presión alta desde valores de $140 \mathrm{mmHg}$ la sistólica y a partir de 90 $\mathrm{mmHg}$ la diastólica (8).

Se estimó como diabético a la persona con diagnóstico médico previo, uso de antidiabéticos por indicación mèdica o cumple con los criterios:

1. glucemia en ayunas mayor o igual a 126

2. Glicemia al azar mayor o igual $200 \mathrm{mg} / \mathrm{dl}$ con síntomas de poliuria, polidipsia y pérdida de peso. 3. Test de tolerancia a la,glucosa mayor o igual a $200 \mathrm{mg} / \mathrm{dl}$.

4. Hemoglobina glicosilada mayor de 6.5\% (9).

El índice de masa corporal IMC es el resultado del Peso en $\mathrm{Kg}$. entre estatura en metros, al cuadrado $(\mathrm{Kg} / \mathrm{m} 2)$, considerando como peso normal (IMC 18.5 a 24.9), sobrepeso (IMC > 25 a 29.9) y obesidad (IMC $>30)(10)$.

\section{PROCEDIMIENTOS}

Se hizo una encuesta realizada por los investigadores en la que se consignaron las variables anteriormente mencionadas. Cada paciente firmó un consentimiento informado en el cual se especificaba toda la información acerca del estudio, aceptando de esta manera su participación. Las variables edad, sexo, tabaquismo y diabetes fueron proporcionados directamente por ellos, mientras que respecto al IMC se solicitó al Servicio de Enfermería información sobre peso y talla para el cálculo del índice de masa corporal. Para la medición de la presión arterial se usó la guía del JNC-7 (8) para lo cual el paciente debe estar en posición sentado con el brazo apoyado, situado a la misma altura que el corazón y sin cruzar las piernas. Se utilizaron los mismos manómetros de mercurio calibrados y de la misma marca en todos 
Ios pabellones, siendo cada tensiómetro calibrado por la Unidad de Ingeniera Clínica del HNAL y validado por expertos.

El modelo de riesgo de Framingham utilizado para establecer el riesgo de desarrollar un evento cardiovascular en 10 años fue el de D Agostino 2008 (12). Clasificados como riesgo bajo: menor a $10 \%$, riesgo moderado: entre 10 y $20 \%$ y riesgo alto: mayor a $20 \%$, se calculó dichos riesgos en el programa Excel por los investigadores.

Participaron encuestadores entrenados, para llenar el formato y para medir la presión arterial.

\section{Aspectos Éticos}

El protocolo y el consentimiento informado fueron aprobados por el Centro de Investigación y Comité de Ética del Hospital Nacional Arzobispo Loayza, así como también por el Centro de Investigación y Comité de Ética de la Facultad de Medicina Humana de la Universidad de San Martin de Porres.

Los datos fueron procesados en el programa estadístico SPSS versión 21. Para realizar los siguientes análisis:

\section{RESULTADOS}

Según el score de Framingham, se encontró que $51.7 \%$ de la población de estudio presentó mediano y alto riesgo para un evento cardiovascular en 10 años. (Figura 2).

El promedio de edad observada fue 54,9 años. Pacientes con diabetes: $22,3 \%$ y con hipertensión arterial sistólica: 15,5\%. El 29,7\% presentó sobrepeso

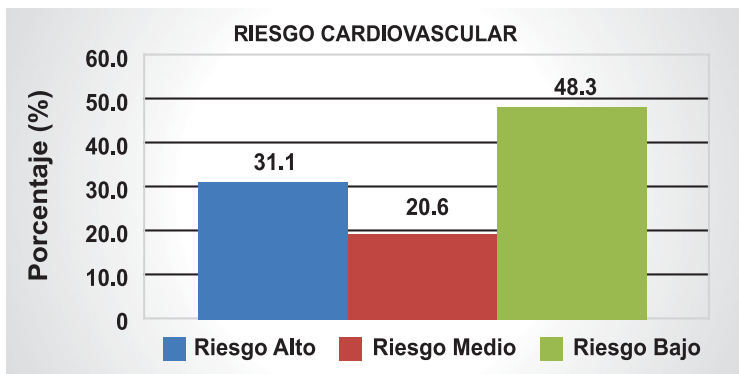

Figura 2. Riesgo Cardiovascular de la población hospitalizada en el Servicio de Medicina Interna- HNAL. Febrero 2014 y un 13,3\% presentó obesidad. La población presentó un riesgo cardiovascular promedio de 16,2 lo cual es clasificado como mediano riesgo. (Tabla 1).

En varones, el riesgo cardiovascular fue de $21,9 \%$ en promedio correspondiendo a riesgo alto, las mujeres presentaron 10,5\% (riesgo moderado). Los hombres, presentaron mayor porcentaje de tabaquismo $(32,8 \%)$ que las mujeres $(11,8 \%)$. La población femenina presentó un porcentaje de diabetes $(29,4 \%)$ mayor que la población masculina $(26,9 \%)$ (Tabla 1).

En cuanto a la edad vascular (EV), la media en la población fue de 61,9 años. El 68,9\% de los pacientes presentó una mayor EV que su edad cronológica (valor delta 6,9). Los varones mostraron una mayor EV en promedio que la edad cronológica (media de 63,9 frente a 54,9 años), al igual que las mujeres (media de 60 frente a 55,5 años). El valor delta fue mayor en los hombres ( 9 años) que en las mujeres (4,5 años) (Tabla1).

Datos presentados como promedio \pm desviación estándar o como promedio y porcentaje (\%).

1. Hipertensión Arterial; presión > de $140 \mathrm{mmHg}$.

2. IMC normal: $18.5-24.9 \mathrm{~kg} / \mathrm{m} 2$

3. IMC sobrepeso: $25-29.9 \mathrm{~kg} / \mathrm{m} 2$,

4. IMC obesidad: mayor o igual a 30 .

5. Delta: edad vascular-edad cronológica.

Al describir los factores de riesgo de la población, según estadíos de riesgo; $68.7 \%$ de los pacientes eran mujeres y tenían bajo riesgo, con edad media de 48,5 años. La edad vascular fue inferior en 0,8 años a la edad cronológica. El $7 \%$ tenía diabetes y $84.3 \%$ no eran fumadores (Tabla2).

En población de mediano riesgo, la mayoría fueron hombres $(55,1 \%)$, con edad media de 57,9 años y edad vascular mayor a su edad cronológica en 10,6 años. $24,7 \%$ tenía diabetes y $79,2 \%$ no eran fumadores (Tabla 2).

En la población de alto riesgo: 75,7\% eran hombres, con edad media de 63,7 años y edad vascular mayor a la edad cronológica con promedio de 16 años. $54.4 \%$ eran diabéticos, $32,4 \%$ fumaban y $35,1 \%$ tenían obesidad (Tabla 2). 
Tabla 1. Medias, frecuencias y porcentajes de los factores de Riesgo del Score de Framingham según género. Servicio de Medicina Interna - HNAL. Febrero 2014

\begin{tabular}{lrrr} 
& \multicolumn{1}{c}{ Varones } & Mujeres & \multicolumn{1}{c}{ Total } \\
\cline { 2 - 4 } Edad (años) & $54.9 \pm 11.9$ & $55.5 \pm 11.3$ & $55.1 \pm 11.6$ \\
Presión Sistólica (mmHg) & $122 \pm 16.0$ & $117.6 \pm 16.2$ & $119.8 \pm 16.2$ \\
Hipertensión arterial sistólica1 (mmHg) & $147(18.5)$ & $144.4(12.6)$ & $145.9(15.5)$ \\
IMC (Kg/m2) & $25.8 \pm 6.1$ & $24.8 \pm 5.7$ & $25.3 \pm 5.9$ \\
Normal2 (\%) & 47 & 57.1 & 51.9 \\
Sobrepeso3 (\%) & 40.3 & 27.8 & 29.7 \\
Obesidad 4 (\%) & 12.7 & 15.1 & 13.9 \\
Diabetes (\%) & 26.9 & 29.4 & 28.2 \\
Tabaquismo (\%) & 32.8 & 11.8 & 22.3 \\
Riesgo (\%) & 21.9 & 10.5 & 16.2 \\
Edad Vascular (años) & 9 & $60 \pm 18.5$ & $61.9 \pm 17.9$ \\
Delta5 (años) & $63.9 \pm 17.0$ & 4.5 & 6.9
\end{tabular}

Tabla 2. Factores de Riesgo Cardiovascular por categorías de riesgo según Score de Framingham en los pacientes del HNAL, Febrero-2014.

$\begin{array}{lrrr} & \text { Bajo riesgo } & \text { Mediano riesgo } & \text { Alto riesgo } \\ \text { Hombres } & 36(31.3) & 27(55.1) & 56(75.7 \%) \\ \text { Mujeres } & 79(68.7) & 22(44.9) & 18(24.3 \%) \\ \text { Edad (años) } & 48.5 \pm 10.9 & 57.9 \pm 7.3 & 63.7 \pm 8.3 \\ \text { PA Sistólica (mmHg) } & 112 \pm 13.5 & 119.3 \pm 11.6 & 132.4 \pm 14.9 \\ \text { PA Diastólica (mmHg) } & 69.2 \pm 10.1 & 71.9 \pm 10.6 & 78 \pm 9.3 \\ \text { IMC (kg/m2) } & 23.8 \pm 4.9 \mathrm{~kg} / \mathrm{m} 2 & 27.1 \pm 7.5 & 26.5 \pm 5.7 \\ \text { Normal } & 22.32(62.6) & 22.6(49) & 22.3(37.8) \\ \text { Sobrepeso2 } & 27.17(29.6) & 27.9(24.5) & 27.1(46) \\ \text { Obesidad3 } & 33.73(7.8) & 35.6(26.5) & 35.1(16.2) \\ \text { Diabetes NO } & 107(93) & 31(63.3) & 33(44.6) \\ \text { Diabetes SI } & 8(7) & 18(24.7) & 41(55.4) \\ \text { Tabaquismo NO } & 97(84.3) & 38(79.2) & 50(67.6) \\ \text { Tabaquismo SI } & 18(15.7) & 11(20.8) & 24(32.4) \\ \text { Edad Vascular (años) } & 60.03 & 63.19 & 79.64 \\ \text { Delta4 (años) } & -0.8 & 10.6 & 16 \\ \end{array}$

Datos presentados como frecuencia y porcentaje (\%) o como promedio \pm desviación estandar.

1 IMC normal: $18.5-24.9 \mathrm{~kg} / \mathrm{m} 2$

2 IMC sobrepeso: $25-29.9 \mathrm{~kg} / \mathrm{m} 2$

3 IMC obesidad: mayor o igual a 30

4 Delta: edad vascular-edad cronológica 


\section{DISCUSIÓN}

Al ser las enfermedades cardiovasculares una de las principales causas de morbilidad y mortalidad en el país, es de suma importancia aplicar modelos de predicción del riesgo de padecer este tipo de enfermedades, para dilucidar los factores que aumentan el riesgo y así poder intervenir precozmente mediante campañas de prevención primaria.

El riesgo cardiovascular se puede calcular mediante diversos scores como el PROCAM o SCORE.

Se eligió el score de Framingham del 2008, por ser referente a nivel mundial, además que es usado en varios países latinoamericanos convirtiéndose en la base de intervenciones de prevención primaria en estas poblaciones, dando buenos resultados (14).

Este score es muy completo ya que predice muerte coronaria y también falla cardiaca, stroke, claudicación intermitente y enfermedad vascular periférica, es decir; muestra un mejor panorama del riesgo cardiovascular.

Aplicando el score de Framingham a la muestra evaluada, se puede apreciar que alrededor del 48.3\% de los pacientes se encuentran catalogados como de bajo riesgo cardiovascular, la cual es la categoría de riesgo más frecuente, sin embargo si se suman las categorías de riesgo medio $(31.1 \%$ y alto (20.6\%) obtenemos un porcentaje de la población más alto, alcanzando un $51.7 \%$.

Entonces, enfocándolo de otra manera tenemos una población cuya mayoría presenta riesgo medio $y$ alto lo cual es un porcentaje mayor al encontrado en nuestro país en los estudios Tornasol I y II publicados por Ruiz Morí (14), en los cuales solo se encontró que solo un $40 \%$ de la población peruana estaba ubicada en estas categorías, esta diferencia podría deberse a que nuestro estudio fue dirigido a personas hospitalizadas, en otras palabras enfermas y quizás más expuestas a factores de riesgo.

La DM constituye uno de los factores esenciales cuando se valora el RCT de una población, no solo por la enfermedad per se, sino por la relación directa que existe entre la hiperglicemia mantenida (de ayunas y postprandial) y la morbimortalidad cardiovascular.

Es uno de los más importantes factores de riesgo aterogénico, y su diagnóstico precoz y control adecuados pueden retardar la aparición de la enfermedad cardiaca, y en relación con lo mencionado, algunos autores plantean la equivalencia de la enfermedad diabética con la cardiopatía isquémica (15).

En nuestro estudio, este fue el factor más prevalente $(28,2 \%)$ lo cual difiere de los estudios Tornasol I y II en los cuales se encontró una prevalencia de 4,4\% y $5,7 \%$ respectivamente. Siendo mayor en mujeres que en varones, lo cual también difiere de los estudios mencionados.

Esto es importante ya que significa que la prevalencia encontrada en nuestro estudio es mayor que la prevalencia encontrada en el país lo cual indica que este es el factor al cual deberían enfocarse posteriores estudios y medidas de prevención en el HNAL.

El tabaquismo, es uno de los mayores problemas de salud que aún no ha podido ser controlado a pesar que la OMS considera que esta es la causa más "prevenible" de enfermedad cardiovascular.

El estudio CARMELA (16) encontró que la prevalencia de tabaquismo en Lima era del $26,6 \%$ siendo mayor en varones, mientras que en nuestro estudio este fue el segundo factor más prevalente con $22,3 \%$. Esto difiere probablemente debido a que la nuestra población no solo estaba conformada por pacientes de Lima sino de todo el Perú.

La HTA es una de las condiciones crónicas más conocidas que incrementan el riesgo cardiovascular. En un estudio publicado por Dueñas y cols (17), se considera la HTA como uno de los tres factores de riesgo de mayor representatividad reportándose un $15,0 \%$ de prevalencia en la población adulta. 
En nuestra población, se presentó en un porcentaje muy similar $(15,5 \%)$ y fue el tercer factor más prevalente.

Con respecto a la edad vascular, un estudio publicado por Masson y cols (18), en población Española, encontró que los hombres tenían un valor delta de edad, mayor que en mujeres (media de 8 frente a 6 años) lo cual concuerda con nuestros resultados. Este dato es la cifra más relevante porque nos habla de cuántos años más tienen las arterias de la persona en relación a su edad cronológica, evidenciando su grado de daño endotelial.

Al ser esta variación mayor en los hombres, nos sugiere que en ellos se necesita aplicar medidas preventivo-promocionales para promover la modificación de su estilo de vida hacia uno más sano.

Finalmente, al ser los pacientes de nuestra población en su mayoría de mediano y alto riesgo, es importante mencionar que los factores de riesgo más frecuentes en estas dos categorías fueron: sexo masculino, edad avanzada (de 57,9 años en adelante), la diabetes y el tabaquismo, lo cual sería importante a tenerlo en cuenta ya que sería óptimo que al encontrar algún paciente hospitalizado con estas características, inmediatamente se les tome los datos necesarios para hallar su riesgo cardiovascular exacto y edad vascular de esta manera poder empezar una tratamiento, sea como prevención primaria en forma de una asesoría para cambio de estilo de vida que incluya dieta y ejercicios o secundaria empleando tratamiento farmacológico en los casos que encontremos alto riesgo.

La idea es que podamos identificar a estos pacientes oportunamente.

En conclusión, los hombres y los pacientes diabéticos tienen un riesgo cardiovascular alto. El score de Framingham debería considerarse como una herramienta de prevención en los hospitales.

\section{REFERENCIAS BIBLIOGRÁFICAS}

1. World Health Organization. Global status report on noncommunicable diseases 2010. Geneva: WHO; 2011.

2. Organización Mundial de la Salud. Estadísticas Sanitarias Mundiales 2014. Ginebra: OMS; 2014

3. Castillo I, Armas NB, Dueñas A, González OR, Arocha C, Castillo A. Riesgo cardiovascular según tablas de la OMS, el estudio Framingham y la razón apolipoproteína B/apolipoproteína A1. Revista Cubana de Investigaciones Biomédicas. 2010; 29:479-88.

4. D’Agostino RB, Sr., Vasan RS, Pencina MJ, Wolf PA, Cobain M, Massaro JM, et al. General cardiovascular risk profile for use in primary care: the Framingham Heart Study. Circulation. 2008; 117(6):743-53.

5. Ruiz E, Segura L, Agusti R. Uso del score de Framingham como indicador de los factores de riesgo de las enfermedades cardiovasculares en la población peruana. Rev Per Card. Setiembre - Diciembre 2012; 38(3) : 1-19

6. Guerreros A, Matsuno A, Ruiz E, Farias C. Guía Nacional de Abordaje al Tabaquismo Perú 2010. 1era Ed; Perú 2010

7. Perk J, De Backer G, Gohlke H, Graham I, Reiner Z, Verschuren M et al Guía europea sobre prevención de la enfermedad cardiovascular en la práctica clínica (versión 2012) .Rev Esp Card. 2012;65:937. e1-e66

8. Joint National Committee on Detection, Evaluation, and Treatment of High Blood Pressure: The Seventh Report of the Joint National Committee on Prevention, Detection, Evaluation, and Treatment of High Blood Pressure (JNC VII)

9. Guías de la asociación americana de diabetes (ADA) para diagnóstico y tratamiento de la Diabetes Mellitus 2014

10. Berrington de Gonzalez A, Hartge P, Cerhan JR, Flint AJ, Hannan L, Maclnnis RJ, et al. Body-mass index and mortality among 1.46 million white adults. N Engl J Med. 2010;363:2211-9

11. Gerin W, Schwartz AR, Schwartz JE, Pickering TG, Davidson KW, Bress J, et al. Limitations of current validation protocols for home blood pressure monitors for individual patients. Blood Press Monit. 2002; 7: 313-318.

12. D'Agostino RB, Vasan RS, Pencina MJ, Wolf PA, Cobain M, Massaro JM, et al. General Cardiovascular Rosk Profile for use in PrimarCare: The Framingham Heart Study. Circulation. 2008;117:743-73 Disponible en: http:// www.framinghamheartstudy. org/ riskfunctions/ cardiovascular-disease/ 10-year-risk. php

13. Icaza G, Núñez L, Marrugat J, Mujica V, Escobar MC, Jiménez AL, et al. Estimación de riesgo de enfermedad coronaria mediante la función de Framingham adaptada para la población chilena. Revista médica de Chile. 2009; 137:1273-82.

14. Ruiz E. Riesgo y prevención cardiovascular. $1^{\circ}$ ed. Lima: Unigraph; 2014.

15. Howard B, Best L, Galloway J, James W, Jones K, Lee E, et al. Coronary heart disease risk equivalence in diabetes depends on concomitant risk factors. Diabetes Care. 2006; 29:391-7. 
16. B M Champagne, E M Sebrié, H Schargrodsky, P Pramparo, C Boissonnet, E Wilson. Tobacco smoking in seven Latin American cities: the CARMELA study. Tob Control [revista en Internet] 2010 [acceso 23 Junio 2014];19(6); 457-462 Disponible en: http:// tobaccocontrol.bmj.com/content/19/6/457.long

17. Dueñas, A. Riesgo cardiovascular total en los trabajadores del Hotel “Meliá Cohíba. Rev Cubana Endocrinol [revista en Internet]. 2008 [acceso 23 Junio 2014]; 19(1); Disponible en : http://scielo. sld.cu/scielo.php?pid=\$1561-29532008000100003\&script =sci_ arttext

18. Masson W, Siniawski D, Toledo G, Vita T, Fernández G, Del Castillo $S$ y cols. Estimación de la «edad vascular» basada en el índice de masa corporal en una población en prevención primaria. Asociación con la aterosclerosis carotídea subclínica. Med Clin (Barc). 2013; 140(6):255-259

\section{Agradecimiento}

A Gabriela Santos Revilla (SOCIEM-USMP), por su colaboración en la interpretación de resultados y diseño final del artículo.
Fuentes de financiamiento

El estudio ha sido financiado por los autores.

\section{Conflicto de interés}

Los autores declaran no tener ningún conflicto de interés.

\section{Correspondencia:}

Jean Carlo Mayta Calderón

Dirección: Calle Las Norias \#108 La Molina

Teléfono: 940153240

Correo electrónico: jean.mayta@gmail.com 